\title{
Estimation des doses au personnel lors d'examens radiologiques complexes au moyen du code $\mathrm{MCNP4B}$
}

\author{
J. PAGES ${ }^{1}$, F. VANHAVERE ${ }^{1}$, L. STRUELENS ${ }^{1}$
}

(Manuscrit reçu le 14 mai 2007, accepté le 27 septembre 2007)

RÉSUMÉ Les distributions de la dose de rayonnement diffusé autour du patient et la dose efficace au personnel lors d'examens radiologiques complexes sont calculées. La propagation du rayonnement et le dépôt d'énergie sont simulés au moyen du code Monte Carlo MCNP4B. Le champ de rayonnement, l'appareil à rayons X, le patient et le radiologue (portant un tablier de plomb) sont modélisés mathématiquement. Un grand nombre de géométries d'irradiation sont simulées et les courbes d'isodoses sont déterminées dans les plans horizontal et vertical. L'influence des divers paramètres (tels que l'énergie et la dimension du faisceau, la taille du patient, la région irradiée, la position et l'orientation du travailleur) sur les doses du personnel est analysée. Des algorithmes publiés combinant les valeurs d'un dosimètre blindé et d'un dosimètre non blindé sont utilisés pour estimer la dose efficace ; un nouvel algorithme fournissant des estimations plus précises de dose est proposé pour plusieurs situations.

ABSTRACT Estimation of staff doses in complex radiological examinations using MCNP4B code. Scatter dose distributions around the patient and staff effective dose have been calculated for complex radiological examinations. Radiation transport and energy deposition was simulated using Monte Carlo MCNP4B code. Radiation field, X-ray equipment, patient and radiologist (wearing a lead apron) were described mathematically. A large number of possible irradiation geometries have been simulated and isodoses curves were obtained on the horizontal and vertical plane. The influence of different parameters (like beam energy and size, patient size, irradiated region, worker position and orientation) on the staff doses have been determined. Published algorithms that combine readings of an unshielded and a shielded dosimeter to estimate effective dose have been applied and a new algorythm was proposed that gives more accurate dose estimates for a wide range of situations.

Keywords: staff dose / Monte Carlo calculations / complex procedures

\section{Introduction}

La protection du personnel médical en radiologie interventionnelle est une partie importante de la radioprotection. Les techniques interventionnelles (angiographie, angioplastie coronarienne, drainages percutanés, etc.) sont de plus en plus utilisées

\footnotetext{
1 SCK•CEN, Centre d'étude de l'énergie nucléaire, Boeretang 200, 2400 Mol, Belgique.
} 
et impliquent des opérations plus complexes et prolongées. Ces procédures requièrent la manipulation d'un cathéter près du faisceau de rayons $\mathrm{X}$, ce qui entraîne une exposition non négligeable du personnel médical. L'irradiation du travailleur est très hétérogène, une grande partie de son corps étant blindée par un tablier de plomb. Il est dès lors difficile d'estimer la dose efficace $(E)$ et diverses approches sont utilisées pour l'estimer en cas de port d'un tablier de protection. Diverses études ont tenté de modéliser l'utilisation d'un tablier de plomb. Cependant, la plupart utilisent des faisceaux parallèles ou des sources ponctuelles au lieu du patient comme source de rayonnement diffusé, et l'intensificateur d'image n'est pas simulé non plus. Dans cette étude, un grand nombre de simulations est présenté pour déterminer la distribution de la dose de rayonnement diffusé autour du patient et la dose efficace du personnel, à partir de valeurs de dosimètres personnels. L'influence de divers paramètres (tels que l'énergie et la dimension du faisceau, la taille du patient, la région irradiée, la position et l'orientation du travailleur) sur les doses du personnel est étudiée. Des algorithmes publiés combinant les valeurs d'un dosimètre blindé et d'un dosimètre non blindé sont utilisés pour estimer la dose efficace ; un nouvel algorithme fournissant des estimations plus précises de dose est proposé pour plusieurs situations.

\section{Matériel et méthode}

Une méthode numérique a été utilisée pour déterminer la distribution de la dose dans le corps du travailleur. La diffusion des rayonnements et le dépôt d'énergie sont simulés au moyen du code MCNP4B (Hendricks, 1997). Le code de calcul MCNP ne calcule pas le transport des photons qu'au-delà de $1 \mathrm{keV}$, signifiant que tout photon d'énergie inférieure à $1 \mathrm{keV}$ dépose son énergie localement. La distribution spectrale des rayons $\mathrm{X}$, telle que déterminée par la tension du tube et la filtration est générée au moyen du logiciel générateur de spectre de Cranley et al. (1997). Les propriétés du champ de rayonnement résultant sont déterminées à l'aide de paramètres tels que la distribution angulaire et spatiale du rayonnement et son spectre d'énergie. L'intensificateur d'image est modélisé par un cylindre de $40 \mathrm{~cm}$ de diamètre, dont le fonctionnement est coupé de manière à constituer un absorbeur de rayonnement absolu. Les corps du patient et du radiologue sont générés à l'aide d'un logiciel générant des modèles anthropomorphiques, le Bodybuilder (White Rock Science, Los Alamos, NM87544). Les équations mathématiques anthropomorphiques sont basées sur le modèle MIRD (Eckerman et al., 1996). Le radiologue est protégé par un tablier de plomb et souvent par un col pour la thyroïde. Six modèles de tabliers sont définis sur la base de modèles actuellement disponibles sur le marché. L'efficacité de la protection pour ces modèles a été analysée, en fonction de la tension du tube de rayons $\mathrm{X}$ et de la position du travailleur dans le champ de rayonnement diffusé. Dans cette étude, on 

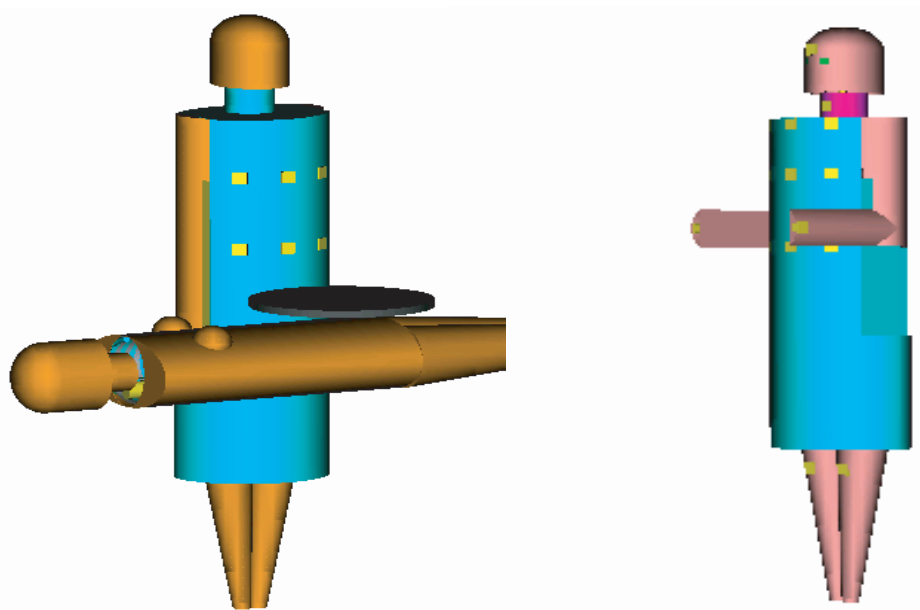

Figure 1 - Les écorchés anthropomorphiques du patient et du personnel.

Patient and staff anthropomorphic phantoms.

ne mentionne que les résultats d'un modèle particulier qui est principalement utilisé : un type long enroulé, $0,5 \mathrm{~mm}$ équivalent plomb sur le devant, $0,25 \mathrm{~mm}$ équivalent plomb dans le dos et sur les côtés, avec un col pour la thyroïde de $0,35 \mathrm{~mm}$ équivalent plomb. Le tablier était modélisé comme la surface d'un cylindre elliptique couvrant le devant, les côtés et le dos du corps représentant le radiologue (des épaules aux genoux). Le tablier ne protège pas les bras; par conséquent, des parties du dos et du devant de la surface cylindrique ont été enlevées et des plaques de liaison en plomb ont été introduites dans le tronc entre les os du bras et les côtes, laissant ainsi les aisselles libres. Les parties avant et arrière du buste sont couvertes par des surfaces elliptiques tronquées. Le col pour la thyroïde est modélisé par une surface cylindrique couvrant le cou. Les avantbras du travailleur sont pliés pour simuler la position des bras lors d'examens cliniques (Fig. 1). Cette situation réaliste des bras pliés influence la dose efficace au travailleur. En général, la dose efficace avec les bras pliés est inférieure à celle avec les bras droits, nos calculs montrant des différences pouvant atteindre les $25 \%$. Au total, 12 dosimètres $H_{p}(10)$ sont placés au cou, au thorax et au niveau de la taille, sur et sous le tablier de plomb. Des dosimètres supplémentaires pour la dose à la peau ont été placés sur le front, les avant-bras et l'avant des cuisses.

\section{Résultats}

Un grand nombre de géométries d'irradiation du patient sont simulées : orientations du tube à rayons $\mathrm{X}$ au-dessus et en dessous de la table et orientations 


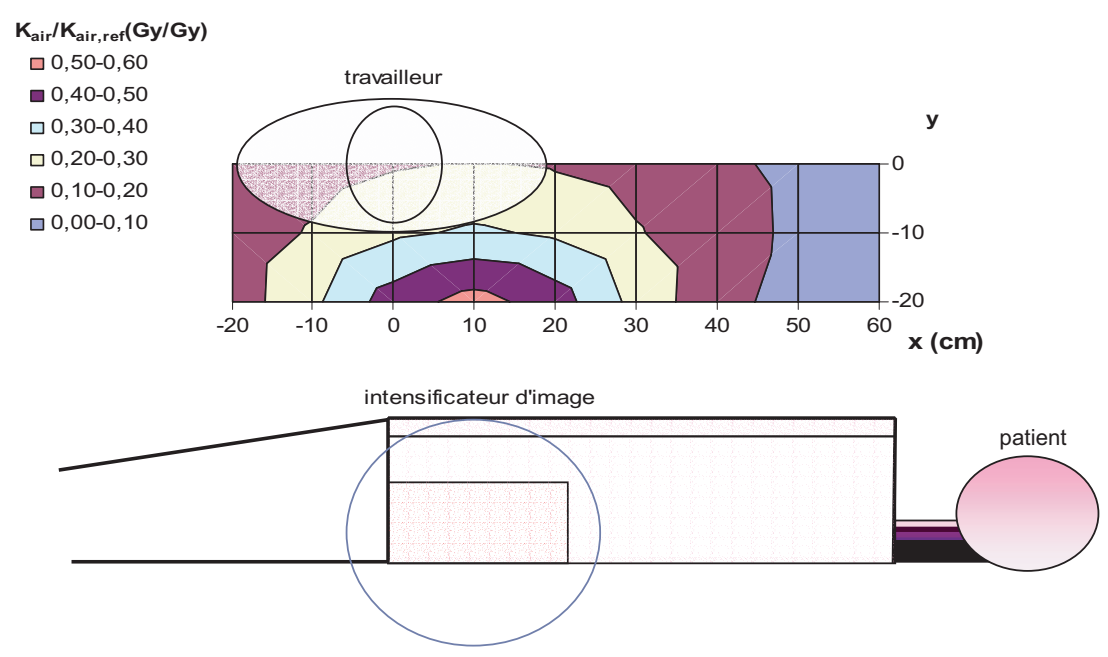

Figure $2-K_{a i r} / K_{a i r, r e f}$ pour l'examen d'une hanche, projection postérieure-antérieure à $76 \mathrm{kV}$. Niveau horizontal.

$K_{a i r} / K_{\text {air,ref }}$ for a pelvis examination, posterior-anterior projection at $76 \mathrm{kV}$. Horizontal plane.

latérales; l'irradiation de la hanche du patient, l'abdomen, le thorax et la tête ; spectres de rayons X de 66 à $96 \mathrm{kVp}$. Les doses de rayonnement diffusé sont calculées dans les trois dimensions : 103 cellules ont été réparties sur une grille tridimensionnelle, à diverses positions, à côté du patient. Pour les différentes géométries, le rayonnement diffusé par le patient est exprimé en kerma dans l'air, normalisé à la valeur la plus élevée, observée d'une irradiation postérieur-antérieur (PA) d'un abdomen avec un faisceau de $76 \mathrm{kVp}$. Les figures 2 et 3 donnent deux exemples au niveau horizontal pour l'examen d'une hanche (postérieur-antérieur, $76 \mathrm{kV}$ ) et l'irradiation d'un abdomen (postérieur-antérieur, $66 \mathrm{kV}$ ). Les valeurs les plus basses normalisées de kerma dans l'air correspondent à l'irradiation de la région du thorax, les valeurs les plus élevées correspondent à l'irradiation de la tête. Pour les projections incidentes postéro-antérieur (under-couch), les doses normalisées $\left(K_{d} / K_{a, r e f}\right)$ sont plus élevées au niveau des jambes du personnel. Pour les projections incidentes antéro-postérieur (over-couch), les doses sont plus élevées au niveau du tronc (Fig. 4). L'erreur relative pour les valeurs de kerma dans l'air s'étend de 0,2 à $17 \%$. On observe les valeurs maxima pour les points de mesure les plus éloignées de la région irradiée.

Dès que le champ de rayonnement diffusé autour du patient est déterminé, les doses aux organes et la dose efficace du travailleur sont calculées. La dose efficace 



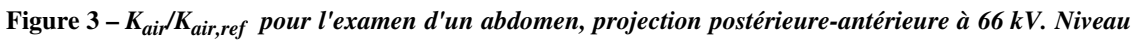
horizontal.

$K_{\text {air }} / K_{\text {air,ref }}$ for an abdominal examination, posterior-anterior projection at $66 \mathrm{kV}$. Horizontal plane.
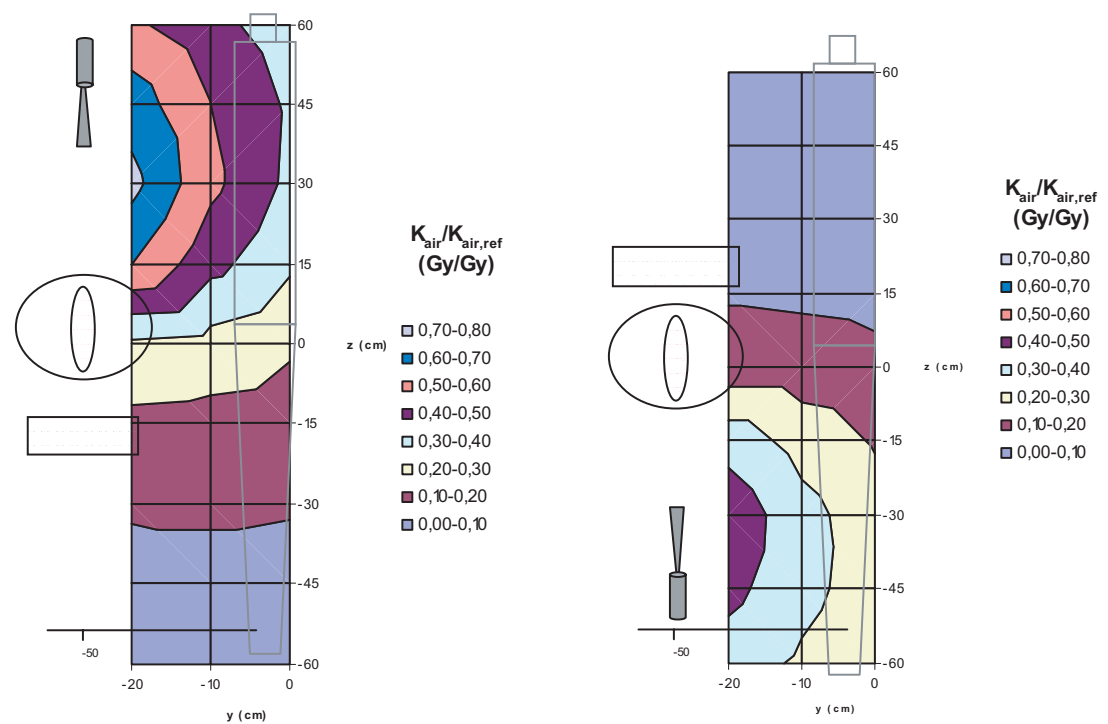

Figure $4-K_{a i r} / K_{\text {air,ref }}$ pour l'examen d'un abdomen, projection antérieure-postérieure et postérieureantérieure à $76 \mathrm{kVp}$. Niveau vertical.

$K_{\text {air }} / K_{\text {air,ref }}$ for an abdominal examination, anterior-posterior and posterior-anterior projections at $76 \mathrm{kVp}$. Vertical plane. 
TABLEAU I

Doses efficaces des travailleurs à diverses positions dans la salle d'opération radiologique, relatives à la valeur PDS.

Effective doses to workers at different positions in the radiological theater relative to the DAP value.

\begin{tabular}{ccccccc}
\hline$E / \mathrm{PDS}\left[\mu \mathrm{Sv} /\left(\mathrm{Gy} \mathrm{cm}^{2}\right)\right]$ & $\mathrm{a}$ & $\mathrm{b}$ (réf) & $\mathrm{c}$ & $\mathrm{d}$ & $\mathrm{e}$ & $\mathrm{f}$ \\
\hline $76 \mathrm{kVp}$ & 0,033 & 0,036 & 0,040 & 0,042 & 0,038 & 0,032 \\
$86 \mathrm{kVp}$ & 0,047 & 0,053 & 0,068 & 0,070 & 0,059 & 0,050 \\
$96 \mathrm{kVp}$ & 0,064 & 0,083 & 0,105 & 0,114 & 0,092 & 0,069 \\
\hline
\end{tabular}

du travailleur est calculée avec la formule suivante :

$$
E=\sum w_{T} w_{R} D_{T, R}
$$

avec $w_{T}$ : facteur de pondération tissulaire, $w_{R}$ : facteur de pondération du type de rayonnement, $D_{T, R}$ : dose absorbée dans l'organe $T$ du rayonnement $R$.

Ces calculs sont effectués (pour chaque champ de rayonnement diffusé autour du patient) pour un maximum de 17 positions différentes du travailleur, chacun avec une distance et une orientation différentes vis-à-vis du patient. Les incertitudes statistiques des doses calculées d'organes dépendent fortement des énergies du photon, du volume et de l'endroit des différents organes. Pour la majorité des doses calculées les erreurs relatives étaient généralement en dessous de $20 \%$. Pour les basses énergies de photons incidents les valeurs simulées pour les petits organes ou les organes plus profonds et totalement protégés par le tablier de plomb, les erreurs respectives sont plus hautes que $20 \%$. La contribution de ces valeurs élevées d'incertitude à la dose efficace est négligeable en raison de la petite contribution de ces organes à la dose efficace. Ceci a comme conséquence des incertitudes relatives moins de $5 \%$ pour les valeurs de dose efficace. Ces doses efficaces du personnel sont calculées, relatives aux valeurs produit dose-surface (PDS). Dans la position de référence, le travailleur se trouve au niveau de l'aine du patient et tourné vers la table (Fig. 2). Sur le tableau I, on voit la dose efficace du travailleur, relative aux valeurs PDS, pour l'irradiation PA de l'abdomen (champ de $18 \times 18 \mathrm{~cm}^{2}$ ). Ces doses sont données pour 6 positions du travailleur en partant d'une position à côté des jambes (a) jusqu'à la tête du patient (f). L'orientation du travailleur est adaptée pour chaque position afin d'avoir des situations réalistes. La position de référence du travailleur est la position b. En général, la dose efficace par PDS du personnel la plus basse fut observée pour l'examen de la hanche, la dose la plus élevée pour les examens de la tête. Les figures 5 et 6 montrent les doses efficaces (relatives à l'irradiation d'un abdomen, $76 \mathrm{kV}$ ) pour des irradiations de l'abdomen, de la hanche, du thorax et de la tête, pour différents 


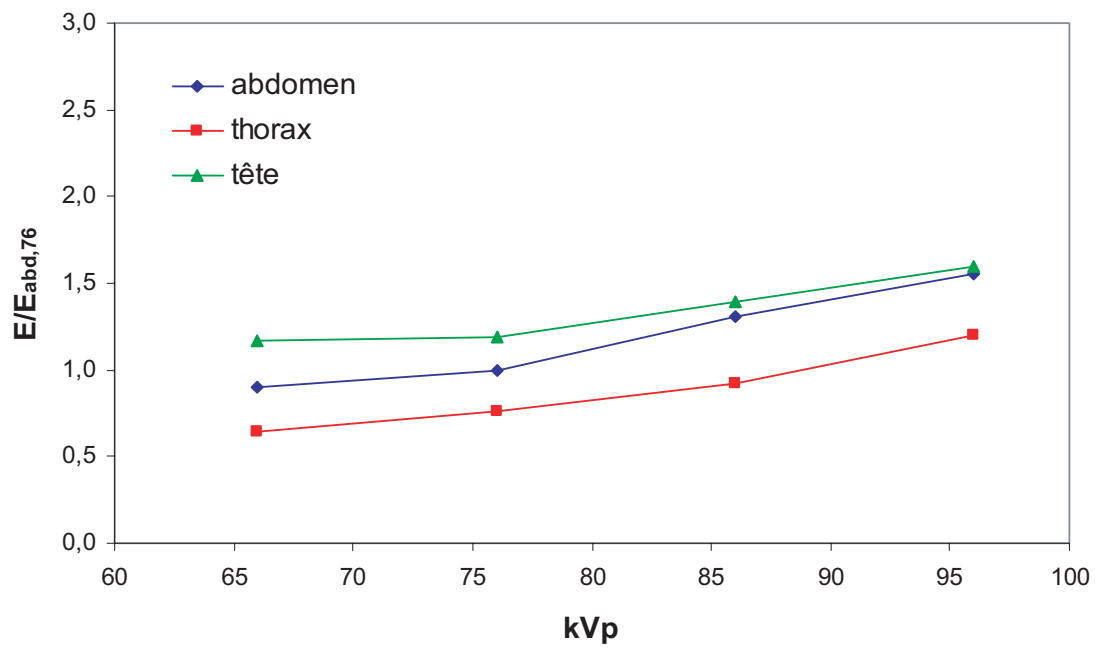

Figure 5 - La figure montre les valeurs normalisées de la dose efficace du travailleur (position b, à côté de l'aine du patient), en fonction de l'énergie du faisceau pour les projections postérieuresantérieures des régions de l'abdomen, du thorax et de la tête du patient. La dose efficace de référence ( $E_{\text {abd76) }}$ correspond à l'irradiation d'un abdomen d'un patient avec un faisceau de rayons $\mathrm{X}$ de $76 \mathrm{kVp}$.

Graph presents worker (position b, near groin of patient) normalised effective dose values in function of beam energy for posterior-anterior projections of the patient abdomen, thorax and head regions. The reference effective dose $\left(E_{a b d 76}\right)$ corresponds to a patient abdomen irradiation with a $76 \mathrm{kVp} X$-ray beam.

$\mathrm{kVp}$. Deux positions du patient ont été sélectionnées : la position de référence b à l'aine du patient, et la position f à la tête du patient.

\section{Discussion}

En bonne logique, l'orientation du tube à rayons $\mathrm{X}$ a une grande influence. La dose efficace du travailleur la plus élevée a été observée pour des irradiations latérales de l'abdomen du patient (Fig. 7, à gauche). Pour la région de la tête, la projection incidente antéro-postérieur présente la dose efficace le plus haute (Fig. 7, à droite). L'écart entre la dose efficace la plus élevée et la plus basse atteint un facteur de 70 pour l'examen de l'abdomen. La géométrie antéro-postérieur engendre des doses 3 à 5 fois plus élevées que celles de la géométrie postéro-antérieur. En ce qui concerne les examens de la tête, l'écart entre la dose efficace la plus élevée et la plus basse atteint un facteur entre 23 à 55. Les géométries antéro-postérieur présentaient des doses qui étaient jusqu'à 6 fois plus élevées que celles des géométries postéro-antérieur. 


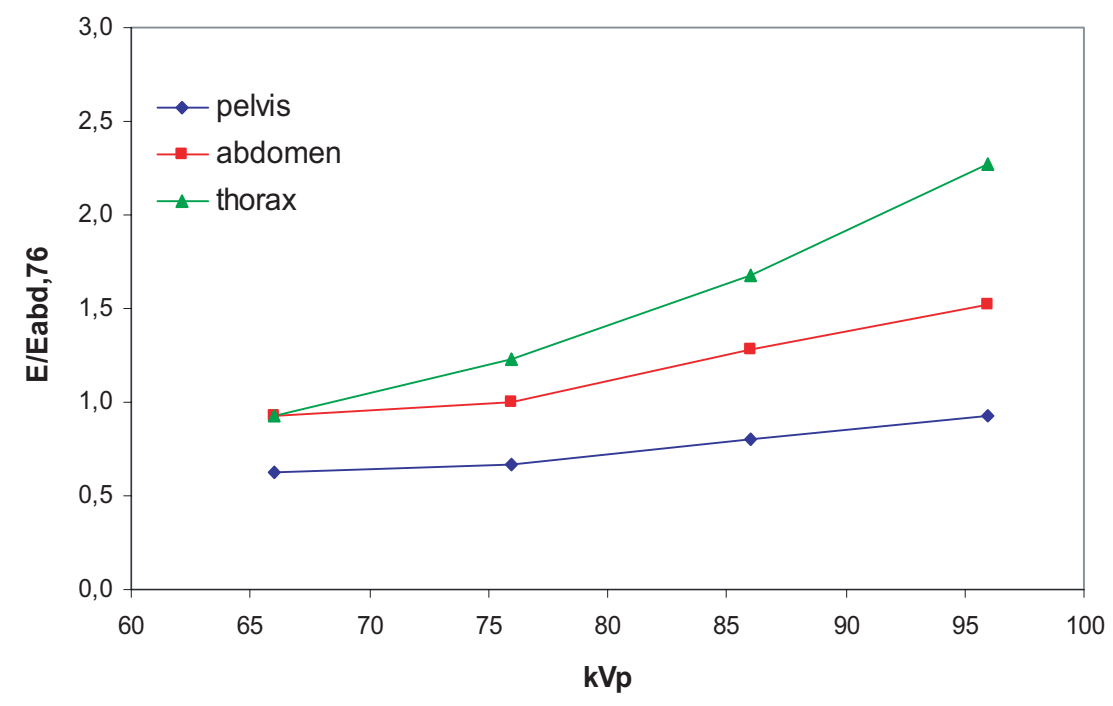

Figure 6 - Le graphique montre les valeurs normalisées de la dose efficace du travailleur (position f, à côté de la tête du patient), en fonction de l'énergie du faisceau pour les projections postérieuresantérieures des régions de la hanche, de l'abdomen et du thorax du patient. La dose efficace de référence $\left(E_{\text {abd76 }}\right)$ correspond à l'irradiation d'un abdomen d'un patient avec un faisceau de rayons $X$ de $76 \mathrm{kVp}$.

Graph presents worker (position $f$, near head of patient) normalised effective dose values in function of beam energy for posterior-anterior projections of the patient pelvis, abdomen, and thorax regions. The reference effective dose $\left(E_{a b d 76}\right)$ corresponds to a patient abdomen irradiation with a $76 \mathrm{kVp}$ X-ray beam.

En tant que paramètre supplémentaire, la dimension du faisceau fut modifiée. Agrandir ou réduire le diamètre du faisceau modifie le volume irradié du patient et par conséquent, la quantité de rayonnement diffusé. La dose efficace est donc proportionnelle à la dimension du faisceau, ce que montre la figure 8 , dans laquelle les doses efficaces pour trois énergies de rayons $\mathrm{X}$ et trois positions du travailleur sont indiquées pour une projection PA de l'abdomen, normalisées à une dimension du faisceau de $24 \times 24 \mathrm{~cm}^{2}$.

La taille du patient a également une influence sur la dose efficace du travailleur. Pour une même tension et un même courant dans le tube, l'intensité du rayonnement diffusé sera plus petite pour les grands patients en raison d'une atténuation plus élevée du faisceau primaire. La dose efficace au travailleur diminue donc lorsque la taille augmente. Cependant, pour la radioscopie il est caractéristique que tant la tension que le courant du tube soient adaptés automatiquement, afin d'obtenir le niveau requis d'exposition d'entrée à 

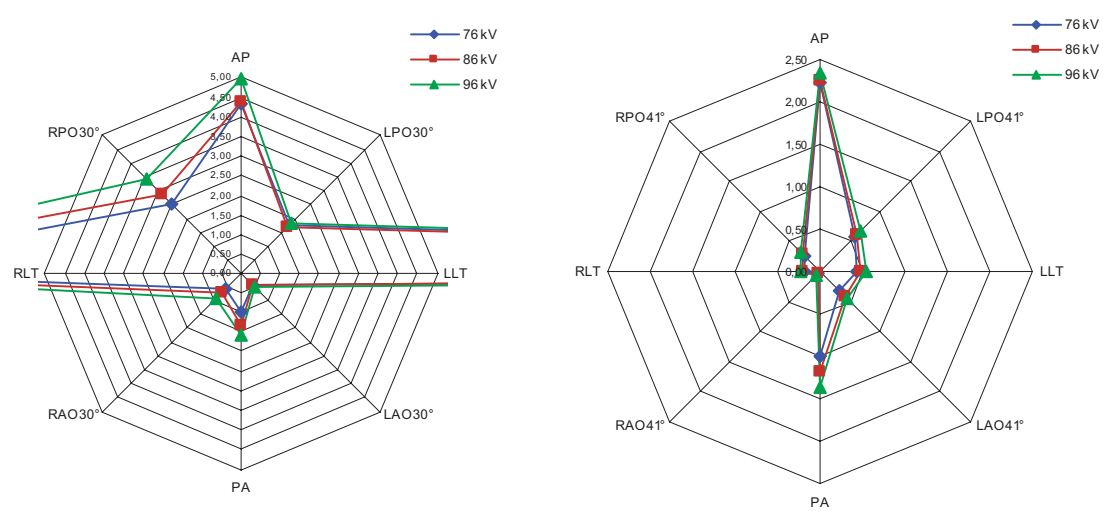

Figure 7 - La dose efficace pour des examens de l'abdomen (gauche) et de la tête (droite). Les valeurs de la dose efficace sont normalisées à la valeur de la dose efficace pour un faisceau de $76 \mathrm{kVp}$, une projection postérieure-antérieure, une position standard du travailleur.

Effective dose for abdomen (left) and head (right) examinations. Effective dose values are normalized to the effective dose value for a $76 \mathrm{kVp}$ beam, posterior-anterior projection, standard position of worker.

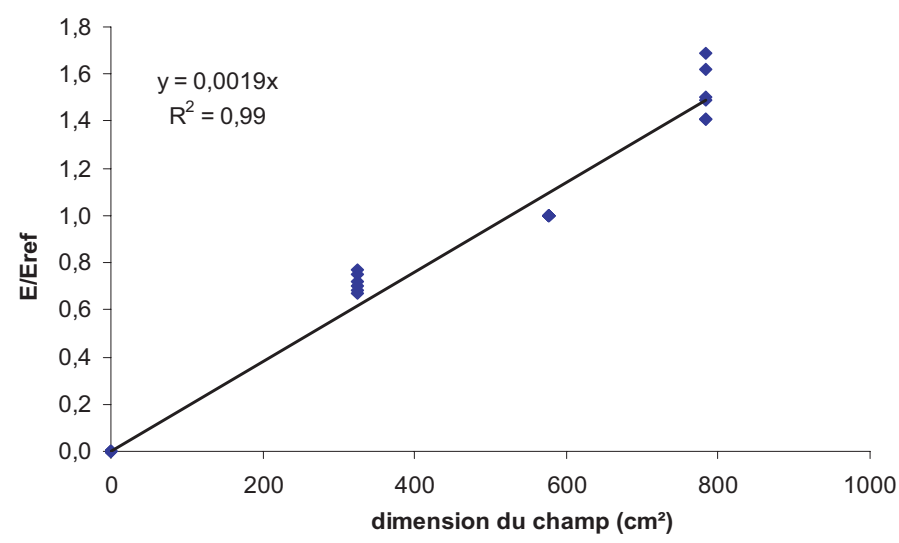

Figure 8 - Le graphique montre les valeurs normalisées de la dose efficace du travailleur, en fonction de la taille du faisceau pour une projection postérieure-antérieure de l'abdomen du patient. La dose efficace de référence $\left(E_{r e f}\right)$ correspond à une taille du faisceau de $24 \times 24 \mathrm{~cm}^{2}$ à la surface d'entrée du patient. Les valeurs normalisées de la dose efficace dépendent de l'énergie du faisceau et de la position du travailleur. Le travailleur porte un tablier enveloppant, de 0,25 mm équivalent de plomb, et un col pour la thyroüde.

Graph presents worker normalised effective dose values in function of beam size for a posterioranterior projection of the patient abdomen. The reference effective doses $\left(E_{r e f}\right)$ corresponds to a $24 \times 24 \mathrm{~cm}^{2}$ beam size at the patient entrance surface. The normalised effective dose values depend on beam energy and worker position. The worker is suited with a $0.25 \mathrm{~mm}$ Pb equivalent wrap-around apron and a thyroid shield. 


\section{TABLEAU II}

L'estimation de la dose efficace du travailleur, à partir des algorithmes disponibles en ce moment. Le rapport de la dose efficace, estimée à l'aide de l'algorithme, à la dose efficace, estimée à partir des simulations Monte Carlo, est présenté pour les irradiations de rayons X de l'abdomen, du thorax, de la hanche et de la tête du patient. Les projections PA, AP, RPO, LPO, RAO, LAO, LR et $L L$ avec faisceaux de $66-96 \mathrm{kVp}$ ont été simulées. Pour les régions du thorax et de la hanche, on n'a simulé que les projections PA. Le travailleur portait un col pour la thyroïde, un tablier enveloppant, de $0,25 \mathrm{~mm}$ équivalent de plomb, et il se trouvait dans les positions RF, RF30, RB et RB78.

Estimation of worker effective dose from currently available algorithms. The ratio of effective dose estimated with the algorithm to effective dose estimated from Monte Carlo simulations is presented for X-ray irradiations of the patient abdomen, thorax, pelvis and head. PA, AP, RPO, LPO, RAO, LAO, LR and LL projections with 66-96 kVp beams were simulated. For the thorax and pelvis regions, only $\mathrm{PA}$ projections were simulated. The worker was wearing a $0.25 \mathrm{~mm} \mathrm{~Pb}$ equivalent wrap-around apron, a thyroid shield and was standing at the RF, RF30, RB and RB78 positions.

\begin{tabular}{llcccc}
\hline \multicolumn{1}{c}{ Auteur } & \multicolumn{1}{c}{ Formule } & \multicolumn{3}{c}{ Rapport $E_{\text {algorithme }} / E_{\mathrm{MCNP}}$} \\
& & Abdomen & Thorax & Hanche & Tête \\
\hline $\begin{array}{l}\text { Huyskens et Franken } \\
(1995)\end{array}$ & $0,033 H_{\mathrm{p}}(10)_{\text {thorax, sur }}+H_{\mathrm{p}}(10)_{\text {taille, sous }}$ & $0,05-1,6$ & $0,2-0,8$ & $0,5-0,7$ & $0,1-0,8$ \\
$\begin{array}{l}\text { Sherbine et De Cicco } \\
(2002)\end{array}$ & $0,07 H_{\mathrm{p}}(10)_{\text {cou, sur }}+H_{\mathrm{p}}(10)_{\text {taille, sous }}$ & $0,06-3,3$ & $0,3-1,1$ & $1,0-1,1$ & $0,1-1,5$ \\
Niklason et al. $(1994)$ & $0,02 H_{\mathrm{p}}(10)_{\text {cou, sur }}+H_{\mathrm{p}}(10)_{\text {taille, sous }}$ & $0,02-0,8$ & $0,1-0,5$ & $0,2-0,5$ & $0,04-0,7$ \\
Wambersie $(1993)$ & $0,1 H_{\mathrm{p}}(10)_{\text {thorax, sur }}+H_{\mathrm{p}}(10)_{\text {thorax, sous }}$ & $0,02-4,6$ & $0,27-1,1$ & $1,2-1,6$ & $0,1-2,2$ \\
NCRP $(1995)$ & $(1 / 21) H_{\mathrm{p}}(10)_{\text {thorax, sur }}$ & $0,01-1,97$ & $0,05-0,34$ & $0,3-0,5$ & $0,03-1,4$ \\
Kicken et al. (1999) & $0,032 H_{\mathrm{p}}(10)_{\text {cou, sur }}$ & $0,01-1,3$ & $0,03-0,2$ & $0,2-0,3$ & $0,02-0,9$ \\
\hline
\end{tabular}

l'intensificateur d'image. Pour les patients de grande taille, un rayonnement primaire plus important est requis afin de satisfaire le fonctionnement de l'intensificateur d'image. L'augmentation du rayonnement primaire entraîne une augmentation du rayonnement diffusé et une augmentation de la dose efficace au travailleur. Dans cette étude, la taille de référence du patient est une femme de $56,5 \mathrm{~kg}$ mesurant 1,68 m. Des simulations supplémentaires sont effectuées pour une femme de $62 \mathrm{~kg}$ et une femme de $68 \mathrm{~kg}$. Les diamètres de leurs troncs étant respectivement 2 et $4 \mathrm{~cm}$ plus grands que celui de la patiente standard. Les doses efficaces normalisées variaient de 1,2 à 1,4 pour la patiente de $62 \mathrm{~kg}$ et de 1,5 à 1,9 pour la patiente de $68 \mathrm{~kg}$, de nouveau en utilisant trois énergies de faisceau et trois positions du travailleur pour une irradiation PA de l'abdomen.

Divers auteurs ont développé des algorithmes pour l'estimation de la dose efficace au travailleur à partir des résultats des badges personnels. Au tableau II, on trouvera un aperçu des algorithmes les plus utilisés. Pour toutes les simulations (133 au total : 66-96 kVp, 5 positions du travailleur, 8 projections différentes) ces algorithmes sont évalués et comparés sur base de la dose efficace. Les algorithmes présents peuvent produire des sous-estimations importantes. 
Un nouvel algorithme a été développé en utilisant les simulations effectuées (133 en total) dans cette étude. Les meilleurs estimateurs linéaires des paramètres de régression sont obtenus par la méthode des moindres carrés.

Ceci signifie que les meilleures valeurs de $\beta_{1}$ et de $\beta_{2}$ sont calculées de sorte que $\varepsilon$ est réduit au minimum dans la formule suivante :

$$
Y=\beta_{1} X_{1}+\beta_{2} X_{2}+\varepsilon
$$

$Y$ est la dose efficace $E$. Pour $X_{1}$ et $X_{2}$ différentes combinaisons des lectures de dosimètre simulées ont été testés. La plus petite variance a été obtenu en employant $H_{\mathrm{p}}(10)_{\text {thorax, sur, milieu devant }}$ et $H_{\mathrm{p}}(10)_{\text {taille, sous, gauche devant. Des valeurs }}$ limites proposées par le NCRP (1995) sont employées, c'est-à-dire $E_{\text {algorythm }} / E_{\mathrm{MCNP}}$ entre 0,9 et 3 . L'algorithme optimum obtenu est :

$$
0,125 H_{p}(10)_{\text {thorax, sur, milieu devant }}+2,4 H_{p}(10)_{\text {taille, sous, gauche devant }} \text {. }
$$

Des projections antéro-postérieur n'ont pas été incluses dans cette formule, parce qu'elles ne sont trouvées que dans de vieux systèmes. Il apparait également que la dose à l'œil peut être estimée en multipliant par 2 la valeur du dosimètre thorax audessus du tablier de plomb. Le rapport du dosimètre du thorax aux doses du cristallin est presque toujours de 0,5 à 1 , et rarement de 1 à 1,5 .

\section{Conclusion}

L'exposition du personnel aux rayonnements dans une salle d'opération radiologique a été simulée de façon réaliste. Plusieurs paramètres sont examinés : l'énergie du faisceau, la région irradiée du patient, la position et l'orientation du travailleur, la taille du faisceau, la taille du patient et la projection du faisceau. Des facteurs de conversion sont calculés pour les doses efficaces pour des différentes positions du travailleur et les valeurs PDS.

Un aspect important est l'utilisation d'un double dosimétrie pour les travailleurs, c'est-à-dire un dosimètre en dessous et un au-dessus du tablier de plomb. L'appropriation des algorithmes disponibles dans la littérature et nos propres algorithmes pour estimer la dose efficace est analysée en les comparant à des données simulées. Le rapport 122 du NCRP (1995) recommande les limites de 0,9 pour la sous-estimation et de 3,0 pour la surestimation lors de la détermination des doses efficaces des travailleurs. Avec cette nouvelle formule, seulement deux situations sur les 133 donnent un ratio légèrement inférieur à 0,9 , alors que trois situations donnent des surestimations de supérieures à 3,0. En limitant la sousestimation à un facteur de 0,9 , la formule fournira généralement des valeurs plus élevées que les algorithmes actuels. 


\section{RÉFÉRENCES}

Cranley K., Gilmore B.J., Fogarty G.W.A., Desponds L. (1997) Catalogue of diagnostique X-ray spectra and other data. Report $\mathrm{N}^{\circ} 78$. York, UK: Institute of Physics and Engineering in Medicine, 1997.

Eckerman K.F., Cristy M., Ryman J.C. (1996) The ORNL mathematical phantom series, http://homer.ornl.gov/vlab/ird.pdf, December 1996.

Hendricks J.S. (1997) MCNP: A general Monte Carlo N-Particle transport code, version 4B, Manual LA-12625-M, Version 4B, Los Alamos, NM: Los Alamos National Laboratory.

Huyskens C.J., Franken Y. (1995) Assessment of effective dose for medical X-ray workers. Proceedings International Conference on Radiation Protection and Medicine. Montpellier, France, 28-30 June 1995.

Kicken P.J., Kemerinck G.J., Schultz F.W., Zoetelief J. (1999) Dosimetry of occupationally exposed persons in diagnostic and interventional arteriography. Part II: assessment of effective dose, Rad. Prot. Dosim. 82, 105-114.

NCRP (1995) National Council on Radiation Protection and Measurements. Use of personal monitors to estimate effective dose equivalent and effective dose to workers for external exposure to lowLET radiation. Bethesda, MD: NCRP report 122.

Nicklason L.T., Marx M.V., Chan H.P. (1994) The estimation of occupational effective dose in diagnositc radiology with two dosemeters, Health Phys. 67, 611-615.

Sherbine S., De Cicco J. (2002) Estimation of the effective dose when protective aprons are used in medical procedures: a theoretical evaluation of several methods, Health Phys. 83, 861-870.

Wambersie A., Delhove J. (1993) Radiation protection in diagnostic radiology, a debated practice: how to wear the individual dosemeters, J. Belge Radiol. 76, 382-385. 